\title{
Editorial:
}

\section{Resident Assessment Instruments: Their Use for Health Care Planning and Research}

Aging populations, especially in industrialized nations, place increased demands on health care services. Because of their diverse and complex health problems, older persons consume a greater proportion of health care services than the young. Rising health care costs attributed largely to older persons, coupled with financial constraints, have become a major focus of concern in Canada and other countries. The challenge for policy makers, regulatory agencies and service providers in resolving this concern is to reduce costs of health care to older persons while maintaining its quality.

The U.S. Institute of Medicine has defined quality of care as "the degree to which health services for individuals and populations increase the likelihood of desired health outcomes and are consistent with current professional knowledge" (Lohr \& Walker, 1990). In 1983 the U.S. Congress asked the Institute of Medicine to examine that country's nursing home system, a major contributor to health care costs, and recommend ways to improve it. One of the Institute's central recommendations was the development of a uniform, comprehensive resident assessment. The articles in this issue illustrate the use of the Resident Assessment Instrument (RAI), which grew out of that recommendation, to address quality of care for older persons.

The RAI was developed by the Health Care Financing Administration (HCFA), the federal agency responsible for setting nursing home standards. The U.S. Congress gave HCFA authority to develop the RAI and regulations governing its use in the Omnibus Budget Reconciliation Act of 1987 (OBRA-87). HCFA's new assessment and regulations were implemented in Spring 1991 and now apply to more than 90 per cent of all nursing homes in the United States.

The nursing home version of the RAI was developed by a multidisciplinary team of health professionals. It includes three key components. The first is a set of assessment items that provide a comprehensive profile of each resident. This component, called the Minimum Data Set (MDS), covers various domains which include: background and customary routines, communication/hearing patterns, physical functioning and structural problems, mood and behaviour patterns, disease diagnosis, oral/nutritional status, skin condition, special treatments and procedures, cognitive patterns, vision patterns, continence, activity pursuit patterns, health conditions, oral/dental status and medication use. The second 
component of the RAI is a set of specialized Resident Assessment Protocols (RAPs) that are intended to link the MDS information to care plan decisions. The RAPs specify additional, highly focussed assessments if a resident's MDS suggests current or potential problems. The third component of the RAI is a user's manual with instructions on how to complete the MDS and RAP assessment procedures and clinical guidelines for developing care plans.

U.S. nursing home facilities must use the RAI to assess residents at admission, upon any significant change in their health status or at least annually. More frequent regular assessments can be performed to monitor the effects of care and any need for modification. Some states also use the RAI data to identify residents with complex care needs or high disability in activities of daily living (ADL). These data are then used to adjust facilities' reimbursement by the Medicaid program, using an algorithm known as the Resource Utilization Group (the RUGS-III system). RAI data are being used for various other purposes, including the development of quality indicators (QIs) based on items in the MDS. For example, QIs in the MDS can provide estimates of the occurrence of resident outcomes such as decline in cognitive status, and of various quality-related events including falls.

The international continuing care community has also found multiple uses for the RAI. Geriatricians, academic researchers and other professionals committed to developing and using standardized assessment tools to improve care for older persons have formed an international organization called interRAI. The nursing home RAI is being actively used in several countries worldwide in appropriate translations, allowing for cross-national comparisons. In addition, members of interRAI have worked to develop adaptations of the RAI for home care, acute care, mental health and post-acute care settings so that clients can now be followed as they move through the continuing care system.

The utility of the nursing home RAI for improving quality of care for elderly residents has been evaluated under the auspices of HCFA in the United States. A pre-test post-test design was used to examine changes in the process of care and longitudinal resident outcomes in a large randomly selected sample of nursing homes. The study observed several positive results, including: an increase in the amount and accuracy of information in residents' medical files, an increase in the comprehensiveness of care planning, improvements in several care processes such as resident and family involvement in care planning, reduction in decline in some areas such as physical and cognitive status, and reduction in resident hospitalization rate without increased mortality (Phillips, Hawes, Morris, Mor, \& Fries, 1996). Studies have shown that the majority of nursing home administrators and staff are positive about the RAI's impact on quality of care, although staff note that it adds to their paperwork burden (Marek, Rantz, Fagin, \& Kreji, 1996). Some facilities have also experienced diffi- 
culties using the RAI properly, which has resulted in inaccurate data (Sgadari et al., 1997). On the whole, however, the RAI seems to be useful for care planning.

Increasingly the RAI is also being used for research purposes. Investigators are examining RAI databases to identify risk and prognostic factors for various health outcomes and to assess the effects of clinical interventions among elderly health care clients across the continuing care system. Proponents of this trend have pointed out that databases have several advantages over traditional ad hoc research (Black, 1997).

Ad hoc studies are often limited in a number of ways. Results may not be widely generalizable because these studies tend to be conducted in a few, possibly atypical settings. Small sample sizes can also limit generalizability through atypical participants, plus restrict statistical power for the comparison of client groups and stratification by client characteristics that might influence interpretation of the results. The rapidity with which change occurs across the continuing care system may mean that the findings of ad hoc research, which is time-consuming to carry out, are irrelevant before they are obtained. Ad hoc research also tends to be expensive so that only a narrow range of studies examining a circumscribed set of risk/prognostic factors or interventions can be mounted. In addition, ad hoc studies may involve overlapping collection of data and place considerable burden on participating clients who find themselves "overstudied". Meanwhile on-site clinicians, with no direct involvement in data collection, may have their routines interrupted with little sense of ownership in the research.

Databases derived from tools like the RAI series are an appealing alternative to the ad hoc research approach because they avoid some of these problems. Databases that are common across the continuing care system make it possible to involve many settings and thus increase generalizability. Large sample sizes can be generated to also increase generalizability and statistical power. Because information is collected on a routine basis, research can be carried out quickly and at less expense. Duplication of effort can be avoided to reduce client burden; for example, several ad hoc studies could use the same database information on physical status so that clients would not need to be measured repeatedly. Various clinicians who normally collect database information could feel more part of the research effort, expanding ownership beyond a small number of clinical researchers directly involved in specific studies.

Despite these advantages, observers have raised some concerns about database research. Foremost, it has been suggested that a comprehensive measure (like the RAI) designed to tap all care-relevant aspects of a client's status is unlikely to address such issues in enough depth to meet research needs (Teresi \& Holmes, 1992). Database proponents counter that both good clinical practice and good research require accurate data (Hawes, Phillips, Mor, Fries, \& Morris, 1992). However, accuracy is not the only 
issue. The presence of information on specific variables required to test a hypothesis and nature of the details available are obviously crucial. Lack of information has been a significant limitation of database research in the past and may remain so. It is difficult to anticipate, if even possible to include due to paperwork demands, all of the variables in a database that may become important to future research. Similarly, measurement tools used to collect database information may become "obsolete" as new tools are developed.

Some concerns have also been raised about the accuracy of database information. Research instruments must be standardized whenever possible but the MDS is unstandardized in important ways (Teresi \& Holmes, 1992). Typically the MDS and its training manual do not specify a standard set of items for collecting data; rather, the manual illustrates how item definitions can be turned into interviewer questions. Consequently, each rater can decide the number of items, which items and the wording to use for resident-specific assessments. In addition, there can be wide variation in which staff complete the MDS, whom they consult as sources (e.g., residents, family, other staff) and the time that they take to complete the task. Measurement variation can seriously affect the validity of data, especially subjective constructs such as behaviour. Reliability can also be affected by variation in the approach to data collection. Such concerns can be raised about some tools used in ad hoc studies, however, so that they are not unique to database research.

Despite concerns that have been raised about the psychometric properties of database information (Ouslander, 1994), concerns that can also be applied to tools used in ad hoc studies, there is increasing evidence that RAI data are psychometrically sound. All RAI instruments were developed through extensive consultation and review, a process which contributes to face and content validity. Convergent validity has been examined by determining whether domains in the instrument are logically correlated, for example, disease diagnosis and medication use (Gambassi et al., 1998). A number of concurrent validity studies have also been conducted; for example, the MDS Cognitive Performance Scale has been shown to correlate at about .80 with the Mini-Mental State Examination (Morris et al., 1994). In addition, there is evidence for the predictive validity of the MDS, with one study showing that MDS fall data on nursing home residents were strong predictors of subsequent falls (Lipstiz et al., 1994).

Reliability studies have also been conducted on the RAI in recent years. U.S.-established inter-rater reliability figures for MDS items range from .40 for some subjective constructs like mood and behaviour to .70 or better for more objective ones like cognitive/decision-making skills or ADL selfperformance (Morris et al., 1997). Cross-national inter-rater reliability figures are similar to one another (Sgadari et al., 1997), as are routinely kept clinical records when compared to the results of research studies (Phillips \& Morris, 1998). There are few summary scales in the MDS, but 
one exception, the Index of Social Engagement, has been shown to have high internal consistency (Mor et al., 1995). Test-retest reliability of the MDS and a related concept, sensitivity of the tool to detect change, have not been specifically addressed, although outcome studies have been able to find changes in resident status.

Comparison studies, within or across countries, using MDS data raise other concerns, some of which, to varying degrees, again apply to ad hoc research. If comparison studies are undertaken between sites, within or across countries, risk adjustment based on client status becomes an important consideration (Berlowitz et al., 1996). For example, it would be inappropriate to compare rate of falls between nursing home settings without adjusting for the risk of falling among their residents based on factors like physical status. Likewise potential subject response bias between sites, such as reporting of pain by different cultures, needs to be considered. Staff may also introduce response bias of various types, which may be aggravated when care planning and reimbursement-related data are used for research. Staff may score residents as having maintained/improved their status to avoid triggering more time-consuming assessments or to appear successful. Conversely they may score residents as having declined to detract from an unpopular program or to maximize funding. These factors complicate not only studies between facilities in the same or different countries but also longitudinal studies within the same facilities whose biases may vary over time.

Nevertheless, carefully developed, computerized databases derived from instruments like the RAI offer an excellent opportunity for research that is valuable to society. RAI data, as illustrated in this issue, can be used to profile client groups, to explore associations among client variables and to conduct non-randomized clinical trials. In this issue, Phillips and Spry describe U.S. nursing home residents with a history of chronic mental illness compared to other residents on demographic characteristics, functional status, behaviours and therapeutic interventions; while Hirdes et al. cross-nationally describe nursing home residents diagnosed with depression and anti-depressant use in Canada, Japan, Iceland and the Czech Republic. Finne-Soveri et al. examine disease diagnosis, physical disability, cognitive impairment and gender as possible predictors of pain among Nordic nursing home residents; while Gilbart and Hirdes consider health, stress, social engagement and social support as possible predictors of psychosocial well-being among residents in Canadian facilities. Gambassi et al. incorporate MDS data into an assessment of the effects of digoxin versus ACE inhibitors versus combination therapy on rate of ADL decline among U.S. nursing home residents with congestive heart failure. Finally, Morris et al. and Bjorkgren et al. respectively address cross-national reliability of the home care RAI in Canada, the U.S., Japan and Italy, and validation of a home care version of the RUGS-III case-mix classification 
system in the U.S. All of these authors have been careful to address potential concerns related to database research in their own studies.

Some of the articles in this issue include Canadian data. In Canada, the RAI has been mandated for use in chronic care hospitals and all long-term care facilities in Ontario and Saskatchewan, respectively. Evaluation studies of the RAI are underway in other provinces, including Alberta. The Alberta site recently developed a new tool, incorporating MDS items, called the Continuing Care Needs Determination Instrument (CCNDI). Thus, RAI databases are becoming increasingly available to Canadian researchers interested in studying health care for older persons.

The benefits of high quality databases for health care planning and research have been recognized for years. The RAI/MDS represents a major accomplishment towards providing a common assessment language across professions and care settings over time. Research conducted using databases should be careful to supplement available information so that specific research questions can be validly answered, to measure site-specific reliability and to address issues such as risk adjustment and response bias. Developments in information technology have made valuable longitudinal health care databases a real possibility. The worth of such databases will be increased if clinicians and researchers collaborate to maximize their design and management.

\section{References}

Berlowitz, D.R., Ash, A.S., Brandeis, G.H., Brand, H.K, Halpern, J.L., \& Moskowitz, M.A. (1996). Rating long term care facilities on pressure ulcer development: Importance of case-mix adjustment. Annals of Internal Medicine, $124,557-563$.

Black, N. (1997). Developing high quality clinical databases. British Medical Journal, 315, 381-382.

Gambassi, G., Landi, F., Peng, L., Brostrup-Jensen, C., Calore, K., Hiris, J., Lipsitz, L., Mor, V., \& Bernabei, R. (1998). Validity of diagnostic and drug data on standardized nursing home resident assessments: Potential for geriatric pharmaco-epidemiology. Medical Care, 36, 22-34.

Hawes, C., Phillips, C.D., Mor, V., Fries, B.E., \& Morris, J.N. (1992). MDS Data should be used for research. The Gerontologist, 32, 563-564.

Lipstiz, L., Nakajima, I., Gagnon, M., Hirayama, T., Conelly, C.M., \& Izumo, H. (1994). Muscle strength and fall rates among residents of Japanese and American nursing homes: An international cross-cultural study. Journal of the American Geriatrics Society, 42(9), 953-959.

Lohr, K.N., \& Walker, A.J. (1990). The utilization and quality of control peer review organization. In Medicare: A strategy for quality assurance. Washington, DC: Institute of Medicine/National Academy Press.

Marek, K.D., Rantz, M.J., Fagin, C.M., \& Kreji, J.W. (1996). OBRA-87: Has it resulted in better quality of care? Journal of Gerontological Nursing, October, 28-36. 
Mor, V., Branco, K., Fleishman, J., Hawes, C., Phillips, C., Morris, J., \& Fries, B. (1995). The structure of social engagement among nursing home residents. Journals of Gerontology: Psychological Sciences, 50, 1-8.

Morris, J.N., Fries, B.E., Mehr, D.R., Hawes, C., Phillips, C., Mor, V., \& Lipsitz, L. (1994). MDS cognitive performance scale. Journal of Gerontology: Medical Sciences, 49(4), M174-M182.

Morris, J.N., Nonemaker, S., Murphy, K., Hawes, C., Fries, B.E., Mor, V., \& Phillips, C.D. (1997). A commitment to change: Revision of HCFA's RAI. Journal of American Geriatrics Society, 45, 1011-1016.

Ouslander, J.G. (1994). Maximizing the Minimum Data Set. Journal of the American Geriatrics Society, 42, 1212-1213.

Phillips, C.D., Hawes, C., Morris, J., Mor, V., \& Fries, B.E. (1996). Effects of the RAI on quality of care: Executive summary. Research Triangle Park, NC: Research Triangle Institute, Program on Aging and Long-Term Care.

Phillips, C.D., \& Morris, J.P (1998). The potential for using clinical and administrative data to analyze outcomes for the cognitively impaired: An Assessment of the Minimum Data Set for Nursing Homes. Alzheimer Disease and Associated Disorders, 11, 162-167.

Sgadari, A., Morris, J.N., Fries, B.E., Ljunggren, G., Jonsson, P.V., DuPaquier, J.-N., \& Schroll, M. (1997). Efforts to establish the reliability of the RAI. Age and Ageing, 26(suppl. 2), 27-30.

Teresi, J.A., \& Holmes, D. (1992). Should MDS data be used for research? The Gerontologist, 32, 148-149. 